\title{
Principal null directions of perturbed black holes
}

\author{
Z. Perjés and M. Vasúth \\ KFKI Research Institute for Particle and Nuclear Physics, \\ H-1525, Budapest 114, P.O.B. 49, Hungary
}

November 9, 2018

\begin{abstract}
The properties of principal null directions of a perturbed black hole are investigated. It shown that principal null directions are directly observable quantities characterizing the space-time. A definition of a perturbed space-time, generalizing that given by Stewart and Walker is proposed. This more general framework allows one to include descriptions of a given space-time other than by a pair $(M, g)$ where $M$ is a four-dimensional differential manifold and $g$ a Lorentz metric. Examples of alternative characterizations are the curvature representation of Karlhede and others, the Newman-Penrose representation or observable quantities involving principal null directions. The conditions are studied under which the various alternative choices of observables provide equivalent descriptions of the space-time.
\end{abstract}

\section{Introduction}

The necessary and sufficient condition for a space-time to be algebraically special in the Petrov classification is 1 that the curvature invariants $I$ and $J$ satisfy

$$
I^{3}-27 J^{2}=0 .
$$

The curvature invariants are the determinantal expressions in the components of the Weyl spinor [2]

$$
\begin{aligned}
& I=\left|\begin{array}{ll}
\Psi_{0} & \Psi_{1} \\
\Psi_{3} & \Psi_{4}
\end{array}\right|+3\left|\begin{array}{ll}
\Psi_{2} & \Psi_{1} \\
\Psi_{3} & \Psi_{2}
\end{array}\right| \\
& J=\left|\begin{array}{lll}
\Psi_{0} & \Psi_{1} & \Psi_{2} \\
\Psi_{1} & \Psi_{2} & \Psi_{3} \\
\Psi_{2} & \Psi_{3} & \Psi_{4}
\end{array}\right| .
\end{aligned}
$$

A Newman-Penrose (NP) notation for the components of a symmetric spinor is used here, i.e.,

$$
\Psi_{m}=\Psi_{0 \ldots 1}
$$


with $m$ the number of spinor indices 1 .

Baker and Campanelli [3] introduce the speciality index of the space-time as the ratio

$$
S=\frac{27 J^{2}}{I^{3}} .
$$

For a Kerr-Newman black hole in the Kinnersley tetrad 4, the only nonvanishing component of the Weyl spinor is $\Psi_{2}$. Expressions (2) then take simple forms and the condition (11) is satisfied (as it should for a type D space-time). Baker and Campanelli go on and expand the speciality index in powers of an arbitrary perturbation parameter $\varepsilon$ of the black hole:

$$
S=1-3 \varepsilon^{2} \frac{\Psi_{0} \Psi_{1}}{\left(\Psi_{2}\right)^{2}}+\mathcal{O}\left(\varepsilon^{3}\right) .
$$

This is a perplexing result since it implies that perturbed black holes, in the first-order approximation, are algebraically special. There exists an extensive literature [5, 6, 7, 8, of 'algebraically special perturbations'. What are these then? It is our purpose in the present paper to shed some light on this apparent controversy by examining the properties of the principal null directions. The clarification of this point leads one to fundamental issues such as the notion of the perturbation of a space-time.

A general framework for perturbations of space-times has been discussed by Stewart and Walker [9]. They propose the following

Definition 1 (Stewart-Walker) A space-time $\left(\mathcal{M}^{\prime}, g^{\prime}\right)$ consisting of the manifold $\mathcal{M}^{\prime}$ and metric $g^{\prime}$ is a perturbation of some given space-time $(\mathcal{M}, g)$ if there exists a smooth one-parameter family of space-times $(\mathcal{M}, g(\lambda))$ in a Hausdorff five-dimensional manifold $\mathbf{M}^{(5)}$ which contains $(\mathcal{M}, g)=(\mathcal{M}, g(0))$ and $\left(\mathcal{M}^{\prime}, g^{\prime}\right)=(\mathcal{M}, g(\varepsilon))$. The unperturbed or background space-time $(\mathcal{M}, g)$ is given by $\lambda=0$. In the parameter interval $\lambda \in(-\varepsilon, \varepsilon)$, the $g^{a b}(\lambda)$ define a piecewise smooth tensor field $g^{\alpha \beta}$ on $\mathbf{M}^{(5)}$ of signature $(0+---)$. The singular hypersurfaces $g^{\alpha \beta}(d \lambda)_{\beta}=0$ are then the original space-time manifolds $\mathcal{M}_{\lambda}$.

A weakness of this definition is that it is too restrictive to be applicable to some important works in perturbation theory. Many treatments of black-hole perturbations characterize the space-time by using curvature components and other quantities which are not considered in the definition. As an example, Chandrasekhar [10] characterizes a perturbed black hole such that the dyad components of the Weyl spinor plus the optical scalars differ only by first-order terms from their form in the background space-time. Such treatments are admissible only in a general framework in which the characterization of the space-time in more than one ways is allowed. For example, in the curvature representation [11, a space-time is locally completely determined by the Riemann tensor and a finite number of its derivatives in a moving frame.

It thus appears necessary to formulate the perturbation problem in a more general way than by the Stewart-Walker definition. If one wants to encompass the various alternative characterizations of the space-time, one may replace the 
pair $\left(\mathcal{M}^{\prime}, g^{\prime}\right)$ in the definition by a pair $\left(\mathcal{M}^{\prime}, \mathbf{O}^{\prime}\right)$ where $\mathbf{O}^{\prime}$ is some complete set of measurable quantities characterizing the space-time. The complete characterization of the geometry is the central issue in what is known the equivalence problem [11, 12.

Definition 2 A set of observables $\mathbf{O}$ on some open set $\mathcal{U}$ is said to locally completely characterize the space-time containing $\mathcal{U}$ if the differentiable and metric structures are fixed uniquely on $\mathcal{U}$ by the values of $\mathbf{O}$.

Obvious examples of the choice of $\mathbf{O}^{\prime}$ are the perturbed metric $g^{\prime}$ or the quantities used in the curvature representation [11]. An incomplete set of observables, for example, in a space-time containing a number of different matter fields is obtained when dropping the subset of observables such as the field stresses of one of the fields from among the observables.

The proposed new definition of a perturbed space-time raises some questions which did not complicate the old definition. An important such question is whether two different choices of $\mathbf{O}^{\prime}$ yield equivalent treatments of the perturbation problem. In the context of linear perturbations, we may formulate this as the requirement that the two choices $\mathbf{O}$ and $\mathbf{O}^{\prime}$ of the observables are related by $\mathbf{O}^{\prime}=M \mathbf{O}$ where $M$ is a nonsingular matrix.

Our goal in this paper is to investigate the sets of observable quantities characterizing the space-time in order to clarify if different choices of these sets of observables will yield equivalent descriptions of the perturbations. In particular, we want to clarify how the various choices of observable quantities behave under perturbations of the system. As a first step on this route, in the next section we recapitulate the behavior of rotational metric perturbations in Hartle's theory [13. In Sec. 3 we briefly recall the concept of principal null directions of a symmetric spinor. The application of this general theory to the electromagnetic and gravitational fields in a Kerr-Newman black hole is reviewed in Sec. 4. The behavior of principal null directions of the electromagnetic vs. gravitational field to first order in the perturbation parameter is worked out in Secs. 5 and 6] respectively.

We find, as an unexpected result of this investigation, that there are certain choices of the sets of observables which provide inequivalent descriptions of the space-time. The existence of these inequivalent sets is shown to have important consequences for the theory of gravitational radiation. This finding, when combined with the properties of principal null directions in the perturbative picture, makes it possible to reach the correct interpretation of 'algebraically special perturbations'.

\section{Illustrative example: rotational perturbations}

In the Hartle theory [13] of rotational perturbations, the metric of the unperturbed vacuum is given by the Schwarzschild solution with mass $m$. The 
perturbed space-time has the metric

$$
\begin{aligned}
d s^{2} & =(1+2 h)\left(1-\frac{2 m}{r}\right) d t^{2}-(1+2 j)\left(1-\frac{2 m}{r}\right)^{-1} d r^{2} \\
& -(1+2 k) r^{2}\left[d \vartheta^{2}+\sin ^{2} \vartheta(d \varphi-\omega d t)^{2}\right] .
\end{aligned}
$$

Here the perturbation functions $h, j, k$ and $\omega$ are assumed to depend smoothly on the perturbation parameter, i.e., the angular velocity $\Omega$. Additional infor-

mation on the form of these quantities is obtained from the discrete symmetries of the rotating system. Taking into account that the simultaneous reversal of time direction and the reversal of the sense of rotation is an exact symmetry, we have that the series expansion of the function $\omega$ contains only odd powers of the angular velocity. The expansion of all other perturbation functions has only even powers in $\Omega$. Hence one finds that the only unknown function in the perturbation problem to first order in $\Omega$ is $\omega$. In fact, the field equations yield an uncoupled, second-order linear differential equation for the function $\omega$. Given the solution of this equation, one may proceed to solve the perturbation problem to second order in the angular velocity $\Omega$. In the second approximation, the field equations are coupled second-order linear differential equations for the remaining perturbation functions. The function $\omega$ contributes quadratic source terms in these equations.

The rotational perturbations of the Schwarzschild black hole illustrate our point: a description which takes into account the full physical properties of the perturbed system can yield restrictions on the form of the series expansion in the perturbation parameter.

\section{Principal null directions}

The principal spinors of a $k$-index symmetric spinor $\varphi_{A B C \ldots K}$ are defined as the nonzero spinors $O^{A}$ satisfying the condition

$$
\varphi_{A B C \ldots K} O^{A} O^{B} O^{C} \ldots O^{K}=0 .
$$

Let $o^{0}$ be the nonzero component and let us introduce the complex ratio

$$
z=\frac{o^{1}}{o^{0}}
$$

The roots of the complex algebraic equation

$$
\varphi_{0}+k \varphi_{1} z+\left(\begin{array}{c}
k \\
2
\end{array}\right) \varphi_{2} z^{2}+\left(\begin{array}{c}
k \\
3
\end{array}\right) \varphi_{3} z^{3}+\ldots+\varphi_{k} z^{k}=0
$$

[where we are using the notation (3) for the components of the spinor] define the flagpoles [14] $o^{A} \bar{O}^{A^{\prime}}$. The principal null directions of $\varphi_{A B C \ldots K}$ are represented, up to real multiplying factors, by these flagpoles. By the fundamental theorem of the algebra, there exist $k$ roots. 


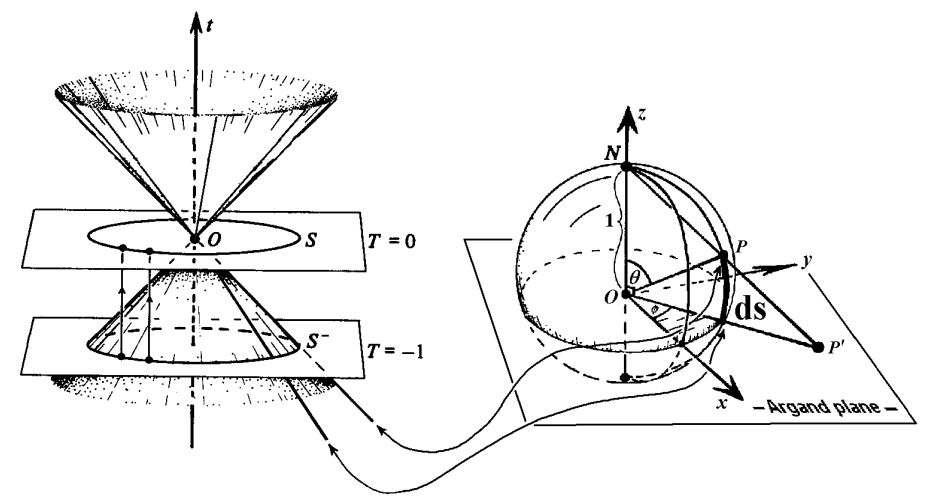

Figure 1: The angle subtended by two principal null directions for an observer is given by the line element ds of his celestial sphere.

The various coincidences of the principal null directions are classified by the partitions of the number $k$. In the generic type, there are $k$ distinct principal directions, represented by $k$ vectors of the light cone in the tangent space $T_{p}$ at point $p$ of the space-time (Figure 1). All other types are called algebraically special.

Principal null directions at any given point are directly observable quantities of the space-time. An observer with world line through the point $p$ can detect principal null directions by looking at a distant sphere [15]. In the lowestorder approximation, the observed image of the sphere is distorted to an ellipse. Kristian and Sachs 16 characterize the distortion by the ratio of the major and minor axes of the ellipse:

$$
D=\frac{1}{2} C(R, \vartheta, \varphi) R^{2}
$$

depending both on the distance $R$ and the direction $(\vartheta, \varphi)$ of the source. To the present accuracy, the distance $R$ can be equivalently taken to be the luminosity distance, the distance by the apparent size or area distance. The coefficient $C(R, \vartheta, \varphi)$ is the projection of the Weyl tensor in the tangent plane of the celestial sphere [16. At the principal null directions, the coefficient $C$ of the distortion has the limiting value $\lim _{R=0} C(R, \vartheta, \varphi)=0$.

The angle subtended by a pair of these directions is given as the invariant length $s$ on the unit sphere $\mathcal{S}^{2}$ with metric

$$
d s^{2}=d \vartheta^{2}+\sin ^{2} \vartheta d \varphi^{2} .
$$

In an orthogonal frame $\{\mathbf{t}, \mathbf{x}, \mathbf{y}, \mathbf{z}\}$, the position of point $p$ of Minkowski spacetime is $T \mathbf{t}+X \mathbf{x}+Y \mathbf{y}+Z \mathbf{z}$. The sphere $\mathcal{S}^{2}$ is represented [14 by the intersection 
of the past null cone with the hyperplane $T=-1$. The polar coordinates $\vartheta$ and $\varphi$ are related to the complex stereographic coordinates $z$ and $\bar{z}$ by the transformation

$$
z=e^{i \varphi} \cot \frac{\vartheta}{2}
$$

The metric of $\mathcal{S}^{2}$ in stereographic coordinates has the form

$$
d s^{2}=\frac{4}{(1+z \bar{z})^{2}} d z d \bar{z} .
$$

The coordinate system $(z, \bar{z})$ is singular at the north pole where $z \rightarrow \infty$, and the coordinate distortion due to the presence of the metric coefficient $P=$ $\frac{1}{2^{1 / 2}}(1+z \bar{z})$ increases indefinitely as one approaches the pole.

There exists a sizable literature of the properties of principal null directions. Penrose and Rindler devote a chapter of their monograph 14 to a detailed study of this subject. Gunnarsen et al. 15] develop a numerical approach, based on the d'Inverno-Russel Clark version of the Ferrari formula, for computing the gravitational principal null directions. They employ this method for the KastorTraschen metrics containing colliding black holes. The status of observations of principal null directions, along with various proposed techniques has been reviewed by Chrobok and Perlick [17].

\section{The Kerr-Newman black hole}

The Kerr-Newman black hole with mass $\mathrm{m}$, rotation parameter $a$ and electric charge e has the metric

$$
\begin{aligned}
d s^{2} & =\left(1-\frac{2 \mathrm{~m} r-\mathrm{e}^{2}}{\zeta \bar{\zeta}}\right)\left(d t-a \sin ^{2} \vartheta d \varphi\right)^{2} \\
& +2\left(d t-a \sin ^{2} \vartheta d \varphi\right)\left(d r+a \sin ^{2} \vartheta d \varphi\right) \\
& -\zeta \bar{\zeta}\left(d \vartheta^{2}+\sin ^{2} \vartheta d \varphi^{2}\right)
\end{aligned}
$$

with the four-potential

$$
A=-\frac{\mathrm{e} r}{\zeta \bar{\zeta}}\left(d t-a \sin ^{2} \vartheta d \varphi\right)
$$

where

$$
\zeta=r-i a \cos \vartheta .
$$

The coordinates $t$ and $r$ run from $-\infty$ to $\infty$ while $\vartheta$ and $\varphi$ are coordinates on a 2 -sphere such that $\varphi$ is periodic with period $2 \pi$ and $\vartheta$ ranges from 0 to $\pi$. In the generic case, the expression

$$
\boldsymbol{\Delta}=r^{2}+a^{2}-2 \mathrm{~m} r+\mathrm{e}^{2}
$$

has two distinct roots. 
The two double gravitational principal null directions in the Kinnersley tetrad are at the south versus the north poles of $\mathcal{S}^{2}$, i.e., at the values of the stereographic coordinates $z=0$ and $z=\infty$. Since the coordinate system is singular at the north pole, it is more advantageous to use the null tetrad [2]

$$
\begin{aligned}
D & \equiv \ell^{a} \frac{\partial}{\partial x^{a}}=\frac{\partial}{\partial r} \\
\Delta & \equiv n^{a} \frac{\partial}{\partial x^{a}}=\frac{1}{2}\left(\frac{2 \mathrm{~m} r-\mathrm{e}^{2}}{\zeta \bar{\zeta}}-1\right) \frac{\partial}{\partial r}+\frac{\partial}{\partial t} \\
\delta & \equiv m^{a} \frac{\partial}{\partial x^{a}}=\frac{1}{2^{1 / 2} \bar{\zeta}}\left[i a \sin \vartheta\left(\frac{\partial}{\partial t}-\frac{\partial}{\partial r}\right)+\frac{\partial}{\partial \vartheta}+\frac{i}{\sin \vartheta} \frac{\partial}{\partial \varphi}\right] \\
\bar{\delta} & \equiv \bar{m}^{a} \frac{\partial}{\partial x^{a}} .
\end{aligned}
$$

In the NP notation, the Maxwell tensor components are

$$
\begin{aligned}
& \Phi_{0} \equiv F_{a b} \ell^{a} m^{b}=0 \\
& \Phi_{1} \equiv \frac{1}{2} F_{a b}\left(\ell^{a} n^{b}+\bar{m}^{a} m^{b}\right)=\frac{\mathrm{e}}{2^{1 / 2} \zeta^{2}} \\
& \Phi_{2} \equiv F_{a b} \bar{m}^{a} n^{b}=\frac{i \mathrm{e} a \sin \vartheta}{\zeta^{3}} .
\end{aligned}
$$

The significant components of the Weyl curvature $C_{a b c d}$ are

$$
\begin{aligned}
\Psi_{2} & =\frac{\mathrm{e}^{2}-\mathrm{m} \bar{\zeta}}{\zeta^{3} \bar{\zeta}} \\
\Psi_{3} & =-3 i a \sin \vartheta \frac{\mathrm{m} \bar{\zeta}-\mathrm{e}^{2}}{2^{1 / 2} \zeta^{4} \bar{\zeta}} \\
\Psi_{4} & =3 a^{2} \sin ^{2} \vartheta \frac{\mathrm{m} \bar{\zeta}-\mathrm{e}^{2}}{\zeta^{5} \bar{\zeta}}
\end{aligned}
$$

and the remaining two components vanish,

$$
\Psi_{0} \equiv-C_{a b c d} \ell^{a} m^{b} \ell^{c} m^{d}=0, \quad \Psi_{1} \equiv-C_{a b c d} \ell^{a} n^{b} \ell^{c} m^{d}=0 .
$$

The curvature singularities are at $r=0$ and $\vartheta=\pi / 2$.

Let the complex ratio of the Maxwell tensor components be denoted

$$
w=\frac{\Phi_{2}}{2 \Phi_{1}}=\frac{i a \sin \vartheta}{2^{1 / 2} \zeta} .
$$

In this notation, the Weyl tensor components satisfy

$$
\Psi_{3}=3 w \Psi_{2}, \quad \Psi_{4}=2 w \Psi_{3} .
$$

The double gravitational principal null directions are at the finite coordinate values $z=0$ and $z=-1 / w$. 
The observation of principal null directions in a Kerr-Newman black hole can be accomplished in an alternative way [18] to the one described in the previous section. There exist special null congruences ('photon trajectories') consisting of the integral curves of principal null directions, characterized by vanishing first curvature $\kappa$ and shear $\sigma$. For these curves, the polar angle $\vartheta$ is constant. The axis value of the angular momentum is $L_{z}=a E \sin ^{2} \vartheta$, with $E$ the conserved energy of the motion, and the Carter separation constant vanishes, $K=0$.

\section{$5 \quad$ Electromagnetic perturbations}

For later reference, we consider here the perturbations of the electromagnetic field in the neighborhood of a black hole. Our first choice for the (incomplete) set of observable quantities is the Maxwell field components. The unperturbed field is given by the components (19). The perturbed Maxwell field has the expansion

$$
\begin{aligned}
& \Phi_{0}=\varepsilon \Phi_{0}^{(1)}+\mathcal{O}\left(\varepsilon^{2}\right) \\
& \Phi_{1}=\Phi_{1}^{(0)}+\varepsilon \Phi_{1}^{(1)}+\mathcal{O}\left(\varepsilon^{2}\right) \\
& \Phi_{2}=\Phi_{2}^{(0)}+\varepsilon \Phi_{2}^{(1)}+\mathcal{O}\left(\varepsilon^{2}\right)
\end{aligned}
$$

where the parenthesized superscripts indicate the degree in the perturbation parameter $\varepsilon$.

The principal null directions of the perturbed Maxwell field are given by the solutions of the quadratic equation

$$
z^{2}+a_{1} z+a_{0}=0
$$

where the coefficients are defined

$$
a_{0}=\frac{\Phi_{0}}{\Phi_{2}}, \quad a_{1}=\frac{2 \Phi_{1}}{\Phi_{2}} .
$$

A possible alternative choice of the observable quantities is the set of principal null directions. We now want to examine if these two choices of the sets of observables are equivalent. The relation between the coefficients $a_{i}$ and the roots $z_{1}$ and $z_{2}$ is given by the Vieté formulae

$$
\begin{aligned}
-a_{1} & =z_{1}+z_{2} \\
a_{0} & =z_{1} z_{2} .
\end{aligned}
$$

Expanding all observable quantities in power series of $\varepsilon$, we have

$$
\begin{aligned}
& a_{0}=\varepsilon a_{0}^{(1)}+\mathcal{O}\left(\varepsilon^{2}\right) \\
& a_{1}=a_{1}^{(0)}+\varepsilon a_{1}^{(1)}+\mathcal{O}\left(\varepsilon^{2}\right) \\
& z_{1}=\varepsilon z_{1}^{(1)}+\mathcal{O}\left(\varepsilon^{2}\right) \\
& z_{2}=z_{2}^{(0)}+\varepsilon z_{2}^{(1)}+\mathcal{O}\left(\varepsilon^{2}\right)
\end{aligned}
$$


where the unperturbed principal null directions are at $z_{1}^{(0)}=0$ and $z_{2}^{(0)}=-1 / w$. Inserting in Eqs. (26), the unperturbed terms cancel and the first-order parts yield

$$
\begin{aligned}
& a_{1}^{(1)}=-\left(z_{1}^{(1)}+z_{2}^{(1)}\right) \\
& a_{0}^{(1)}=z_{1}^{(1)} z_{2}^{(0)} .
\end{aligned}
$$

From these relations we see that the set $\left(z_{1}^{(1)}, z_{2}^{(1)}\right)$ of first-order observables is linearly equivalent to the set $\left(a_{0}^{(1)}, a_{1}^{(1)}\right)$. Our conclusion is that the field stresses of the perturbed Maxwell tensor yield a description equivalent with the perturbed principal null directions.

\section{Gravitational perturbations}

In the literature of perturbed black holes [10, the sets of observable quantities characterizing the gravitational field are selected from several available options. The most frequent two choices are the dyad components of the Weyl spinor in the NP formalism, and the components of the perturbed metrics. These two descriptors of the state are related to each other by the second-order differential equations embodying the field equations and the gauge conditions. Rather than investigating these complicated relations, we want to compare here the two choices of the observables given by the dyad components of the Weyl spinor and by the principal null directions. These alternatives are connected by the quartic equation

$$
\Psi_{4} z^{4}+4 \Psi_{3} z^{3}+6 \Psi_{2} z^{2}+4 \Psi_{1} z+\Psi_{0}=0 .
$$

For the unperturbed black hole, the Weyl spinor components have the form (20) and the four solutions for the principal null directions pairwise coincide: $z_{i}^{(0)}=(0,0,-1 / w,-1 / w)$. We introduce the normalized coefficients

$$
a_{i}=\left(\begin{array}{c}
4 \\
i
\end{array}\right) \frac{\Psi_{i}}{\Psi_{4}}
$$

for $i=0, \ldots, 4$. As in the previous section, we expand all observables of the perturbed space-time in powers of the perturbation parameter $\varepsilon$,

$$
a_{k}=a_{k}^{(0)}+\varepsilon a_{k}^{(1)}+\mathcal{O}\left(\varepsilon^{2}\right) \quad \text { for } k=0, \ldots, 3 .
$$

The two sets of observable quantities are now related by the symmetric expressions

$$
\begin{aligned}
& -a_{3}=\sum_{i=1}^{4} z_{i}, \quad a_{2}=\sum_{i, k=1, i<k}^{4} z_{i} z_{k} \\
& -a_{1}=\sum_{i, k, l=1, i<k<l}^{4} z_{i} z_{k} z_{l}, \quad a_{0}=\prod_{i=1}^{4} z_{i} .
\end{aligned}
$$


Inserting here the series expansions of the roots

$$
\begin{aligned}
& z_{1}=\varepsilon z_{1}^{(1)}+\mathcal{O}\left(\varepsilon^{2}\right) \\
& z_{2}=\varepsilon z_{2}^{(1)}+\mathcal{O}\left(\varepsilon^{2}\right) \\
& z_{3}=-\frac{1}{w}+\varepsilon z_{3}^{(1)}+\mathcal{O}\left(\varepsilon^{2}\right) \\
& z_{4}=-\frac{1}{w}+\varepsilon z_{4}^{(1)}+\mathcal{O}\left(\varepsilon^{2}\right)
\end{aligned}
$$

we get the linear relations for the first-order observables

$$
\begin{aligned}
& a_{0}^{(1)}=0 \\
& a_{1}^{(1)}=\frac{1}{w^{2}}\left(z_{1}^{(1)}+z_{2}^{(1)}\right) \\
& a_{2}^{(1)}=-2 w a_{1}^{(1)}-\frac{1}{w}\left(z_{3}^{(1)}+z_{4}^{(1)}\right) \\
& a_{3}^{(1)}=-\left(z_{1}^{(1)}+z_{2}^{(1)}+z_{3}^{(1)}+z_{4}^{(1)}\right) .
\end{aligned}
$$

Hence we see that the choice of the principal null directions as the set of observables is inequivalent to choosing the set of Weyl spinor components. By Eq. (34), for space-times with perturbed principal null directions, the coefficient $a_{0}$ is perturbed only at the second order in the parameter $\varepsilon$. The second-order contribution, however, is nonvanishing and has the form $a_{0}^{(2)}=\frac{1}{w^{2}} z_{1}^{(1)} z_{2}^{(1)}$.

Here we have an example of a situation when two choices of the sets of observable quantities yield inequivalent characterizations of the perturbed space-time. We then need to decide which of these should be used in the definition of the perturbed space-time. A significant portion of the existing literature uses the Weyl tensor components for characterization, with no regard to the behavior of the alternative set of observables such as the principal null directions. We argue, however, that two space-times having finite (or of lower-order) differences in some of their observable quantities should not be considered as exhibiting small perturbations. In the above situation, the two black hole space-times that have first-order differences of the curvature component $\Psi_{0}$ do not satisfy the linear relation (34), hence they are inequivalent in the sense that they must have their corresponding principal null directions pointing at finite angles.

The correct interpretation of the above situation can be inferred from the behavior of rotational perturbations. When one choice of the set of observables gives rise to space-times which are characterized by finite differences in certain observables, then this indicates that pairs of corresponding quantities with infinitesimal differences will only change in a higher-order approximation with the appropriate choice of the perturbation parameter. For rotational perturbations, one might carelessly expand the diagonal components of the metric with respect to some parameter (such as the square of the angular velocity) such that the perturbed state has first-order contributions in the diagonal metric. That this is not the right choice of the parameter, becomes clear when considering the behavior under reflections. 


\section{Gauge choices}

The foregoing discussion did not extend to the problem of choosing the tetrad gauge. In the exact description of a space-time, the choice of a null tetrad vector along a principal null direction leaves only a small discrete group of gauge symmetries permuting the principal directions. In the present section we show that the gauge group in the linear approximation is much larger, and in fact it has an important geometrical content.

The perturbed tetrad is chosen such that the spinor $o^{A}$ is one of the four principal spinors of the Weyl curvature:

$$
\Psi_{0}=0 \text {. }
$$

For the unperturbed black hole, two independent choices for the spinor $o^{A}$ are possible, corresponding to the two distinct double principal directions.

In perturbation theory, the solutions of (38) remain, to some extent, undetermined in the linear approximation. Rather than having a finite number of exact solutions of Eq. (38), there exist infinitely many solutions of the problem. This can be seen from the expansions (35) of the symmetric expressions. Given the coefficients $a_{i}^{(1)}$, these equations have an infinite number of solutions for the roots $z_{i}^{(1)}$. The multitude of the principal null directions in the linear approximations gives rise to a group of dyad transformations which leaves condition (38) unchanged.

The quantity $\Psi_{0}$ transforms under the infinitesimal dyad transformation

$$
o^{A} \rightarrow o^{A}+b \iota^{A}, \quad \iota^{A} \rightarrow \iota^{A},
$$

as follows:

$$
\Psi_{0} \rightarrow \Psi_{0}+4 b \Psi_{1} .
$$

Here $b$ is an arbitrary but small complex multiplier function such that higher powers of $b$ are negligible. Since the curvature quantity $\Psi_{1}$ itself is small, the spinor $o^{A}$ remains a principal spinor of the curvature under the transformations (391).

Gauge symmetries are normally considered as mathematical properties of our description. A gauge transformation is expected to alter such quantities of the description as the tetrad frame or a field potential while leaving the physically measurable quantities (the curvature or the field strength) invariant. From this point of view, the above setup where we choose a tetrad along a principal null direction is somewhat unusual. Here a gauge transformation of the type (39) amounts to selecting a congruence of principal null curves. The particular congruence thus selected is that with a tangent spinor $o^{A}$. Thus a 'gauge change' of the form (39) amounts to picking a new family of principal null curves. These families of curves may have different propagation properties. Hence the choice of the 'gauge' has significant consequences for the resulting behavior in the linear approximation.

One of the earliest contributions to the literature of algebraically special perturbations by Couch and Newman [5] takes advantage of an especially simple 
form of the metric, and finds the perturbations of a Schwarzschild black hole. The linear algebraically special perturbations of the Kerr black hole are considered by Wald [6] in the NP approach. Wald uses a gauge in which $\Psi_{0}=\Psi_{1}=0$. For this particular pick of the principal null congruence, both the shear and the first curvature vanish. Wald's aim is to prove that also $\Psi_{4}$ vanishes, in order to establish that the knowledge of $\Psi_{0}$ alone suffices for a full description of the perturbed space-time. He fails to achieve this goal for modes with certain 'algebraically special' frequencies. Note that one should proceed with caution when using the results of this paper. For example, on p. 1457, one finds this claim: 'i.e., we obtain the linearized version of the Goldberg-Sachs theorem'. Now the Goldberg-Sachs theorem has been shown not to hold in the linearized theory [19.

Chandrasekhar further investigates the properties of algebraically special perturbations [7]. He concludes his work with several, apparently technical, but at any rate, unanswered questions. These concern the relation of the Starobinsky operator to complex conjugation, and to the special forms of the potential barriers occurring. These questions (he writes) are sheated in enigmas. A detailed investigation of the algebraically special frequencies is to be found in [8].

Despite the deceptive simplicity achieved by the choice of the principal null congruence with $\Psi_{1}=0$, this approach neither offers a complete description of the space-time nor can it be easily generalized for the presence of charge.

\section{Second-order perturbations}

In Section 6, we have shown that the component $\Psi_{0}$ of the curvature does not receive a contribution in the linear approximation. In the light of existing earlier literature of black hole perurbations, it is an important question what are the equations governing the first nonvanishing contribution to the field $\Psi_{0}$.

Our goal in this section is to obtain an uncoupled differential equation for the second-order function $\Psi_{0}$. To this end, we express the derivatives $\delta \rho, \delta \kappa$, $D \tau, D \beta, D \mu$ and $D \bar{\alpha}$ from the NP equations (4.2k), (4.2b), (4.2c), (4.2e), (4.2h) and from the complex conjugate of (4.2d), respectively. Similarly, we express $D \Psi_{2}, \delta \Psi_{2}, D \Psi_{1}$ and $\delta \Psi_{1}$ from the NP electrovacuum Bianchi identities (A3). Where necessary, second derivatives are untangled by use of the commutator $[D, \delta]$. Acting with the commutator $[D, \delta]$ on $\Psi_{1}$, the resulting equation has the form

$$
\begin{aligned}
& {[\delta \bar{\delta}-D \Delta-4 \alpha \delta+2 \bar{\alpha} \bar{\delta}+(4 \gamma-\mu) D+(\bar{\rho}+4 \rho) \Delta} \\
& \left.-8 \alpha \bar{\alpha}+4 D \gamma-4 \delta \alpha-4 \bar{\rho} \gamma-16 \gamma \rho+4 \mu \rho+2 \Psi_{2}\right] \Psi_{0} \\
& =-4(\bar{\delta}-6 \alpha)\left(\sigma \Psi_{1}\right)+4(\Delta-\bar{\gamma}+\bar{\mu}-5 \gamma)\left(\kappa \Psi_{1}\right)-10 \Psi_{1}^{2} .
\end{aligned}
$$

To second order, the terms in the square bracket on the left can be taken to have their value in the Kerr-Newman metric since they act on the second-order quantity $\Psi_{0}$. The terms on the right contain either of the second-order quantities $\sigma \Psi_{1}, \kappa \Psi_{1}$ or $\Psi_{1}^{2}$. The operators acting on these second-order quantities assume 
their values at the Kerr-Newman metric:

$$
\begin{aligned}
\square_{2} \Psi_{0} & =2\left\{-\frac{\sqrt{2}}{\zeta}\left[i a \sin \vartheta\left(\frac{\partial}{\partial r}-\frac{\partial}{\partial t}\right)+\frac{\partial}{\partial \vartheta}-\frac{i}{\sin \vartheta} \frac{\partial}{\partial \varphi}+\frac{3 \cos \vartheta}{\sin \vartheta}\right] \sigma\right. \\
& \left.+\left[\left(\frac{\mathrm{m}(\zeta+\bar{\zeta})-\mathrm{e}^{2}}{\zeta \bar{\zeta}}-1\right)\left(\frac{\partial}{\partial r}+\frac{1}{\zeta}\right)+2 \frac{\partial}{\partial t}-6 \frac{\mathrm{m} \bar{\zeta}-\mathrm{e}^{2}}{\zeta^{2} \bar{\zeta}}\right] \kappa-5 \Psi_{1}\right\} \Psi_{1}
\end{aligned}
$$

where

$$
\begin{aligned}
\square_{s} & =\boldsymbol{\Delta}^{-s} \frac{\partial}{\partial r} \boldsymbol{\Delta}^{s+1} \frac{\partial}{\partial r}+\frac{1}{\sin \vartheta} \frac{\partial}{\partial \vartheta} \sin \vartheta \frac{\partial}{\partial \vartheta}+s\left(1-s \frac{\cos ^{2} \vartheta}{\sin ^{2} \vartheta}\right) \\
& +\left[2 a\left(\frac{\partial}{\partial t}-\frac{\partial}{\partial r}\right)+\frac{1}{\sin ^{2} \vartheta}\left(\frac{\partial}{\partial \varphi}+2 i s \cos \vartheta\right)\right] \frac{\partial}{\partial \varphi} \\
& +a^{2} \sin ^{2} \vartheta \frac{\partial^{2}}{\partial t^{2}}-2\left[\left(r^{2}+a^{2}\right) \frac{\partial}{\partial r}+(s+2) r+i a \cos \vartheta\right] \frac{\partial}{\partial t}
\end{aligned}
$$

is the wave operator introduced in 20 .

Given the first-order solution of the perturbation problem, we have an uncoupled linear differential equation for the unknown function $\Psi_{0}$. The right-hand side is fully known and is to be treated as a source term.

In the second approximation, the issue of the gauge choice arises again. This has been investigated in [21] and [22].

\section{Conclusions}

We have argued above that perturbations of a black hole can be described in a sense which conforms to generally accepted criteria if the lowest-order contributions to the curvature component $\Psi_{0}$ are of second order in the perturbation parameter. First-order contributions to $\Psi_{0}$ would result in finite changes in the principal null directions, which are directly accessible to observations. Before these disturbing results can be considered as fully consolidated, there is a clear need to clarify a number of related issues. We conclude this work with raising just one such example.

The curvature component $\Psi_{4}$ remains invariant under transformations (39). Under infinitesimal dyad transformations of the form

$$
\iota^{A} \rightarrow \iota^{A}+c o^{A}, \quad o^{A} \rightarrow o^{A},
$$

with $c$ an arbitrary small complex function, $\Psi_{4}$ transforms

$$
\Psi_{4} \rightarrow \Psi_{4}+4 c \Psi_{3}
$$

In the Kinnersley tetrad, the spinor $\iota^{A}$ is chosen, as is $o^{A}$, to be a principal spinor for the Kerr metric [4, and both $\Psi_{3}$ and $\Psi_{4}$ vanish. Repeating the argument with the symmetrical expressions (35) for $\Psi_{4}^{(1)}$ and projective coordinate $z^{\prime}=$ $-1 / z$, at first sight it would seem to be possible to choose the null tetrad for 
the perturbed space-time such that the only non-vanishing tetrad component of the Weyl spinor is again $\Psi_{2}$. This would imply that the perturbed space-time is again the Kerr black hole with trivial parameter changes.

To find ways out from controversies like the one just described, either one has to give up insisting on the observability of principal null directions, or else to carry out a careful investigation of perturbations in a neighborhood of the coordinate singularity on the two-sphere. We do not see any ground, however, on which the observability of principal null directions should be given up.

\section{Acknowledgments}

Correspondence on various aspects of this work with colleagues is herewith acknowledged. We thank Hisaaki Shinkai for literature on observational techniques, Lior Burko for sharing his views on the algebraic type, Amos Ori for advice on the scaling of the Weyl tensor components under perturbations, and Sergio Dain on the Goldberg-Sachs theorem. This work has been supported by the OTKA grant T031724.

\section{References}

[1] M.M.D. Kramer, M.A.H. MacCallum, H. Stephani and E. Herlt: Exact Solutions of Einstein's Field Equations (Cambridge University Press, Cambridge, 1980).

[2] E.T. Newman and R. Penrose, J. Math. Phys. 3, 566 (1962), to be referred to as NP.

[3] J. Baker and M. Campanelli, Phys. Rev. D62, 127501 (2000).

[4] W. Kinnersley, J. Math. Phys. 10, 1195 (1969).

[5] W.E. Couch and E.T. Newman, J. Math. Phys. 14, 285 (1973).

[6] R.M. Wald, J. Math. Phys. 14, 1453 (1973).

[7] S. Chandrasekhar, Proc. Roy. Soc. A392, 1 (1984).

[8] A.M. van den Brink, Phys. Rev. D62, 064009 (2000), gr-qc/0001032 preprint (2000).

[9] J.M. Stewart and M. Walker, Proc. R. Soc. London A341, 49 (1974).

[10] S. Chandrasekhar: The Mathematical Theory of Black Holes (Clarendon Press, 1983, p. 430).

[11] A. Karlhede, Gen. Rel. Gravitation 12, 693 (1980).

[12] M. Bradley, in Relativity Today, Eds. C.A. Hoenselaers and Z. Perjés (Akadémiai Kiadó, 2002). 
[13] J. Hartle, Astrophys. J. 150, 1005 (1967).

[14] R. Penrose and W. Rindler: Spinors and Space-Time, Sec. 8 (Cambridge University Press, Cambridge, 1986).

[15] L. Gunnarsen, H. Shinkai and K. Maeda, Class. Quantum Grav. 12, 133 (1995), gr-qc/9406003 preprint (1994).

[16] J. Kristian and R.K. Sachs, Astrophys. J. 143, 379 (1965).

[17] T. Chrobok and V. Perlick, Class. Quantum Grav. 18, 3059 (2001).

[18] C. Misner, K.S. Thorne and J.A. Wheeler: Gravitation (Freeman, 1973).

[19] S. Dain and O.M. Moreschi, J. Math. Phys. 41, 6296 (2000), gr-qc/0203057 preprint (2002).

[20] Z. Perjés and M. Vasúth, Astrophys. J. 582, 342 (2003).

[21] M. Campanelli and C.O. Lousto, Phys. Rev. D59, 124022 (1999).

[22] M. Bruni, S. Mattarrese, S. Mollerach and S. Sonego, Class. Quantum Grav. 14, 2585 (1997), gr-qc/9609040 (1996). 